\title{
New Physics Signals in Longitudinal Gauge Boson Scattering at the LHC
}

\author{
Tao $\mathrm{Han}^{a}$, David Krohn ${ }^{b}$, Lian-Tao Wang ${ }^{b}$, and Wenhan Zhu ${ }^{b}$ \\ ${ }^{a}$ Department of Physics, University of Wisconsin, Madison, WI 53706 \\ ${ }^{b}$ Department of Physics, Princeton University, Princeton, NJ 08544 \\ than@hep.wisc. edu, dkrohn@princeton. edu, lianwang@princeton. edu, wenhanz@princeton. edu
}

\begin{abstract}
We introduce a novel technique designed to look for signatures of new physics in vector boson fusion processes at the $\mathrm{TeV}$ scale. This functions by measuring the polarization of the vector bosons to determine the relative longitudinal to transverse production. In studying this ratio we can directly probe the high energy $E^{2}$-growth of longitudinal vector boson scattering amplitudes characteristic of models with non-Standard Model (SM) interactions. We will focus on studying models parameterized by an effective Lagrangian that include a light Higgs with non-SM couplings arising from $\mathrm{TeV}$ scale new physics associated with the electroweak symmetry breaking, although our technique can be used in more general scenarios. We will show that this technique is stable against the large uncertainties that can result from variations in the factorization scale, improving upon previous studies that measure cross section alone.
\end{abstract}

Keywords: Beyond Standard Model, Higgs Physics, Hadronic Colliders. 


\section{Contents}

1. Vector Boson Fusion as a Probe of New Physics 1

2. Theoretical Setup 3

3. Leptonic and Semi-Leptonic Channels Revisited: Scale Uncertainties 4

4. Polarization Measurements $\quad 7$

4.1 Leptonic Polarization 8

$\begin{array}{lll}4.2 & \text { Hadronic Polarization } & 10\end{array}$

$\begin{array}{lll}4.3 & \text { Scale Uncertainty } & 11\end{array}$

$\begin{array}{lll}4.4 \text { Background } & 12\end{array}$

5. Future Directions 13

5.1 Improving Hadronic Reconstruction 13

$\begin{array}{lll}5.2 & \text { Additional Applications } & 14\end{array}$

6. Conclusions $\quad 15$

A. Scattering Amplitudes for Longitudinal Gauge Bosons and Partial Wave $\begin{array}{ll}\text { Unitarity } & 16\end{array}$

$\begin{array}{lr}\text { B. Overview of Jet Algorithms } & 18\end{array}$

\section{Vector Boson Fusion as a Probe of New Physics}

The Large Hadron Collider (LHC) was built to elucidate the physics behind electroweak symmetry breaking (EWSB). In a sense, it must succeed in finding some new physics because the partial wave amplitudes for $V_{L} V_{L} \rightarrow V_{L} V_{L}$ scattering, ${ }^{1}$ calculated in the absence of a Higgs or other new physics, begin to violate unitarity at the $\mathrm{TeV}$ scale. Therefore, either new weakly-coupled light particles must come in to unitarize the amplitudes, or we will see new strong interactions in the electroweak sector.

While many models of EWSB have been proposed, precision experiments such as LEP seem to favor a model employing a $\mathcal{O}(100) \mathrm{GeV}$ scalar with the quantum numbers and approximate couplings of the Standard Model (SM) Higgs [1, 2]. Many models of new physics already include such a particle, oftentimes with couplings deviating slightly from those of the SM, e.g. little Higgs [3] and holographic Higgs models [4]. Ideally, such models

\footnotetext{
${ }^{1} \mathrm{By} V_{L}$ we denote a longitudinally polarized electroweak vector boson.
} 
would be identified and studied at the LHC through the production of their intrinsic new particles. However, the finite energy reach and large backgrounds at the LHC could make discovering any new states very difficult.

Thus we will focus on these non-SM light Higgs scenarios, both because they are favored by precision data and because they are perhaps the most difficult to distinguish from the SM. To study these setups we will take a model-independent approach, employing an effective field theory to parameterize the effects of new physics $[5,6,7,8,9,10]$. We will see that the general phenomenology of the Higgs sector is captured by the coefficients of a small number of dimension- 6 operators $[11,12]$, only one of which is relevant to the vector boson fusion process we wish to study.

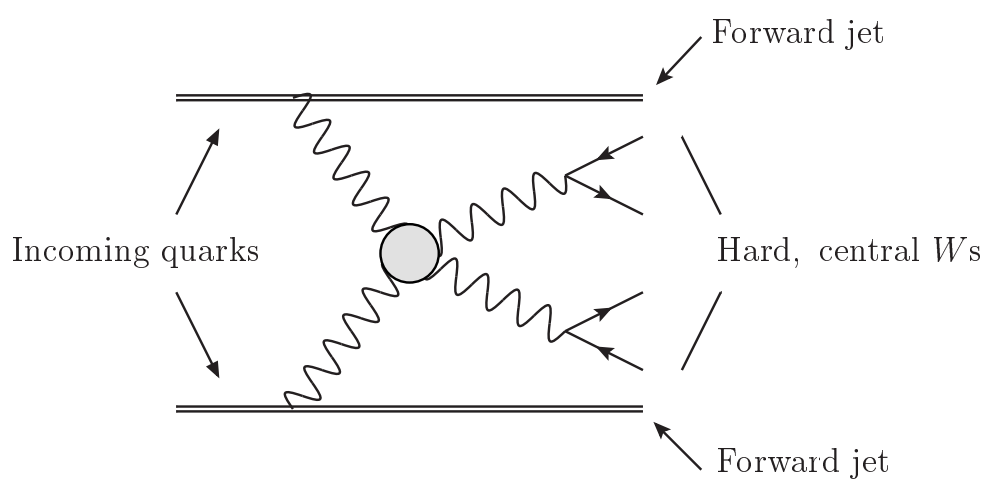

Figure 1: Illustration for vector boson fusion.

Vector boson fusion (VBF) is the process in which vector bosons radiated by initial state quarks scatter into vector bosons (see Fig. 1). This process is intimately tied to EWSB: just as the pion is a Nambu-Goldstone boson (NGB) and $\pi \pi$ scattering can be used to understand chiral symmetry breaking, at high energies longitudinally polarized vector bosons take on the behavior of the NGBs from EWSB. In the absence of a Higgs boson or other new physics responsible for the EWSB, the scattering amplitudes probed by VBF would violate perturbative unitarity $[13,14,15,16]$ at around $1 \mathrm{TeV}$ (see the discussion in appendix A). Furthermore, if the Higgs boson does not have the exact couplings to vector bosons as predicted by the SM, then the necessary cancelations will not occur and one will still observe an $E^{2}$ growth in the amplitudes until new physics comes into play. It is by measuring this growth that we can hope to observe the effects of physics beyond the SM, even in scenarios where we only see a light Higgs-like particle $[12,17]$.

In this article we will introduce a novel technique designed to analyze VBF processes and observe the $E^{2}$ growth in longitudinal gauge-boson scattering amplitudes mentioned above. We will begin by introducing our notations and framework in Section 2. To motivate our new technique, in Section 3 we will update past analyses of VBF (specifically [18] and [19]) taking into account the effects of parton showering and jet clustering. We will show that these analyses, which infer the $E^{2}$ amplitude growth from cross section increases, carry large $\mathcal{O}(100 \%)$ uncertainties due to factorization-scale ambiguities that affect 
jet tagging and vetoing. ${ }^{2}$ Then, in Section 4 we will describe our technique designed to reduce these uncertainties by measuring the relative production of transverse to longitudinal modes, focusing on the fully reconstructable semi-leptonic decay of the $V V$ system. We will demonstrate that this measurement is sensitive to anomalous Higgs-gauge couplings while at the same time being robust against the scale uncertainties that challenge cross section measurements. In Section 5 we will discuss potential improvements in the analysis and comment on other applications of the technique. Section 6 contains our conclusions.

\section{Theoretical Setup}

In the formulation of a general effective theory of the SM-like Higgs sector $[5,6,11]$ most of the operators are tightly constrained $[7,8,9,10]$ because of their otherwise excessive contributions to the electroweak observables, such as the $\rho$-parameter, oblique parameters, and triple gauge boson self-interactions. There are only two dimension-six operators that are genuine interactions in the Higgs sector not subject to the current experimental constraints, $\partial^{\mu}\left(H^{\dagger} H\right) \partial_{\mu}\left(H^{\dagger} H\right)$ and $\left(H^{\dagger} H\right)^{3}$. We note that as both operators are composed from the singlet operator $H^{\dagger} H$ they may serve to probe not only EWSB physics, but also other physics beyond the SM. For a given theoretical framework, the coefficients of these operators may be calculable [12], and by measuring them we can hope to learn about any new physics. Even in some strongly coupled models for which these may not be calculable, the measurement of a non-zero value can give important clues to the structure of new physics. Now, the second operator above does not have derivative couplings, so its effect on the behavior of the $V_{L}$ scattering amplitudes at high energies should be sub-leading [11]. We therefore focus on the former and parameterize it (following [12]) as

$$
\mathcal{L} \supset \frac{c_{H}}{2 f^{2}} \partial^{\mu}\left(H^{\dagger} H\right) \partial_{\mu}\left(H^{\dagger} H\right)
$$

where the coefficient $c_{H}$ is naturally of $\mathcal{O}(1-4 \pi)$ depending on whether the underlying theory is weakly or strongly coupled, and $f$ is the characteristic scale of new physics, typically expected to be round $4 \pi v$ if the new physics is associated with EWSB.

Upon expanding around the electroweak VEV $v$, this operator contributes terms which add to the kinetic terms of $H$. After imposing canonical normalization on the fields, the result is a modification to the Higgs couplings. Ref. [12] parameterizes the resulting modified Higgs-gauge coupling in the zero-momentum limit as

$$
g_{\mathrm{eff}}=\frac{g_{\mathrm{SM}}}{\sqrt{1+c_{H} \xi}} \approx g_{\mathrm{SM}}\left(1-\frac{c_{H}}{2} \xi\right)
$$

where $\xi=v^{2} / f^{2}$. This modified coupling has important phenomenological consequences because it deviates from the SM prediction. At high energies and for $\left|c_{H} \xi\right| \gtrsim 0.1^{3}$ this

\footnotetext{
${ }^{2}$ While one might expect to eventually overcome these uncertainties through a combination of datadriven calibration and higher order calculations, the analysis we describe herein avoids them altogether.

${ }^{3}$ For smaller values of $\left|c_{H} \xi\right|$ the dominant non-SM effects enter as interference terms proportional to $c_{H} \xi$ rather than $\left(c_{H} \xi\right)^{2}$. Also, in this case the anomalous energy dependence of the longitudinal cross section goes as $E^{2}$ instead of $E^{4}$.
} 
modification leads to an incomplete cancelation in the amplitude for longitudinal vector boson scattering and the cross section grows as

$$
\sigma\left(V_{L} V_{L} \rightarrow V_{L} V_{L}\right) \approx\left(\frac{c_{H}}{2} \xi\right)^{2} \sigma\left(V_{L} V_{L} \rightarrow V_{L} V_{L}\right)_{\text {no-higgs }}
$$

which can be seen by considering the NGB scattering as shown in appendix A. In what follows we will study means of measuring this behavior. Note that, as discussed in appendix A, the $W_{L}^{+} W_{L}^{-}$scattering amplitudes calculated in this framework violate perturbative unitarity when

$$
s_{W W} \approx \frac{16 \pi v^{2}}{c_{H} \xi\left(1-\frac{c_{H} \xi}{4\left(1+c_{H} \xi\right)}\right)} .
$$

This is the point at which we expect new physics to come into play. In what follows we will limit our analyses to

$$
\sqrt{s_{V V}}<2 \mathrm{TeV}
$$

This corresponds to a coupling value $\left|c_{H} \xi\right| \sim 0.6$. We will take this as an upper limit for our analyses. Of course, looking beyond this energy range would be interesting and should be attempted at the LHC, but any deviation from the SM expectation would no longer carry the same effective Lagrangian interpretation. Also, note that for larger couplings and lower scales of new physics some higher dimensional operators could become relevant and it would be more appropriate to think of the $c_{H} \xi$ used in our analysis as parameterizing a new physics form factor, rather than as the coefficient of a particular operator.

\section{Leptonic and Semi-Leptonic Channels Revisited: Scale Uncertainties}

The most straightforward way to probe the behavior of Eq. (2.3) would be to measure the resulting increase in VBF cross section at higher energies. This is a well studied topic, with many different analyses having been performed (see, for instance, [18, 19, 20, 21, 22, $23,24,25,26,27,28,29,30,31])$. Here we will revisit these analyses taking into account the effects of parton showering. We will see that while the cuts developed in past analyses remain essential in suppressing background and isolating VBF signals, one encounters large factorization scale uncertainties that affect rate measurements and must be overcome to detect new physics in VBF.

Sophisticated acceptance cuts have been developed to suppress the SM background and isolate the longitudinal gauge boson scattering in VBF processes at high energies. It has been a common practice to impose a high $p_{T}$ cut on the reconstructed gauge bosons or their decay products, require one or two forward (backward) energetic jets, and demand that the central detector region remain relatively free of hadronic activity. The first few cuts ensure that we observe hard scattering processes with the gauge bosons emitted by energetic quarks $[32,33,34]$, while the last cut is designed to reduce background by taking advantage of the fact that VBF is a purely electroweak process with no color exchange [35] and $V_{L} V_{L}$ scattering tends to produce fewer central jets than other electroweak processes [21]. Using this sort of cut, it was concluded $[18,19]$ that reasonable sensitivity can 
be achieved for $\mathrm{TeV}$ scale strongly interacting new physics at the $14 \mathrm{TeV}$ LHC with an integrated luminosity of $100 \mathrm{fb}^{-1}$.

We revisit the $W W$ analyses with the theoretical framework as discussed in the previous section. We consider both fully leptonic [18] and semi-leptonic [19] decays of the vector bosons. For the sake of illustration, we concentrate on the $W^{+} W^{-}$final state. Our VBF parton-level results are generated using the full $2 \rightarrow 6$ matrix element for

$$
q q^{\prime} \rightarrow q q^{\prime} W^{+} W^{-} \rightarrow q q^{\prime} \ell^{ \pm} \nu f \bar{f}^{\prime}
$$

without making the effective $W$ approximation [36, 37, 38]. In so doing, wherever appropriate, we have included other $\mathcal{O}\left(\alpha_{E W}^{6}\right)$ processes as background to the channels. Our PDFs are those of MRST2004 [39]. To generate the jet-level samples we shower partonlevel results using Pythia 6.4.21 [40] with a virtuality ordered shower, cluster the visible final state particles into $0.1 \times 0.1 y-\phi$ cells between $-5 \leq y \leq 5$, and produce $R=0.7$ anti- $k_{T}$ [41] jets using FastJet [42]. To sample PDFs and shower our results we must choose a factorization scale for the gauge boson scattering processes. The natural choice of the factorization scale is of the order of $m_{W}$, with corrections from the $p_{T}$ of the scattering quarks. We parameterize the choice of scales via

$$
\mu^{2}=\beta^{2}\left(m_{W}^{2}+\frac{1}{2} \sum_{\text {jets }} p_{T}^{2}\right),
$$

where $\beta$ is an $\mathcal{O}(1)$ parameter.

We begin by adopting the selection cuts of [18] to study the fully leptonic $W^{+} W^{-}$final state, as detailed in Table 1. Using these cuts, we calculate the parton level cross sections for a light Higgs scenario ${ }^{4}$ with various anomalous couplings parameterized by $c_{H} \xi$. The parton level results for a few representative scale choices of $\beta$ are listed on the left-hand side of Table 2. They are consistent with those of [18]. At this level, the uncertainty in rate is only around $\mathcal{O}(10 \%)$, which can be attributed entirely to the PDFs. When we include showering, hadronization and jet clustering, the scale $\mu$ dictates the virtuality at which the parton shower is started, in addition to controlling the sampling of PDFs. On the right-hand side in Table 2, we present the cross sections for the showered and clustered results with a few representative scale choices. We see that the uncertainties can now be as much as $\mathcal{O}(100 \%)$. This is because small changes in $\mu$ result in large changes in the behavior of the associated forward jets. A higher value of $\mu$ could lead to harder radiation that will sink forward jets below the tagging criteria, or it could lead to the parton-shower emission of a veto jet. As the uncertainties from varying the scale $(\beta=0.5-2.0)$ would normally set the systematic theoretical errors, such large uncertainties in rate would make it difficult to distinguish the presence of anomalous couplings, even for large values of $c_{H} \xi$.

We next explore the situation for the semi-leptonic mode of $W^{+} W^{-}$decay. We employ cuts inspired by [19] as shown in Table 3. The results of this analysis are shown in Table 4,

\footnotetext{
${ }^{4}$ Here and henceforth, we will take a light Higgs boson mass as $m_{H}=100 \mathrm{GeV}$ for illustration and for comparing with the early studies in the literature. This will make no numerical difference with other $m_{H}$ values as long as it is well below the $2 m_{W}$ threshold.
} 


\begin{tabular}{|c|c|}
\hline Leptonic Cuts & Jet Cuts \\
\hline$|y(l)|<2.0$ & $E\left(j_{\text {tag }}\right)>0.8 \mathrm{TeV}$ \\
$p_{T}(l)>100 \mathrm{GeV}$ & $3.0<\left|j_{\text {tag }}\right|<5.0$ \\
$\Delta p_{T}(l l)>440 \mathrm{GeV}$ & $p_{T}\left(j_{\text {tag }}\right)>40 \mathrm{GeV}$ \\
$\cos \phi_{l l}<-0.8$ & $p_{T}\left(j_{\text {veto }}\right)>30 \mathrm{GeV}$ \\
$M(l l)>250 \mathrm{GeV}$ & $\left|y\left(j_{\text {veto }}\right)\right|<3.0$ \\
\hline
\end{tabular}

Table 1: The cuts of [18] for the leptonically decaying $W^{+} W^{-}$final state. The signal selection requires that we tag at least one jet $\left(j_{\text {tag }}\right)$ and to veto extra central jets $\left(j_{\text {veto }}\right)$.

\begin{tabular}{|c|c|c|c|c|c|c|}
\hline & \multicolumn{3}{|c|}{ Parton Level [fb] } & \multicolumn{3}{c|}{ Jet Level [fb] } \\
\hline$c_{H} \xi$ & $\beta=0.5$ & $\beta=1.0$ & $\beta=2.0$ & $\beta=0.5$ & $\beta=1.0$ & $\beta=2.0$ \\
\hline 0.4 & 0.015 & 0.013 & 0.012 & 0.015 & 0.009 & 0.005 \\
0.2 & 0.013 & 0.011 & 0.010 & 0.013 & 0.006 & 0.004 \\
0.0 & 0.011 & 0.090 & 0.008 & 0.012 & 0.007 & 0.004 \\
\hline
\end{tabular}

Table 2: Cross sections [fb] for VBF with $W^{+} W^{-}$final states decaying into $e$ and $\mu$ for various anomalous Higgs-gauge couplings and at different factorization scales parameterized by Eq. (3.2). The cuts used to generate these results are those of [18]. The set of cross sections on the left are computed at parton level, while those on the right correspond to results after the parton shower and hadronization.

again demonstrating a relatively stable signal at the parton level (left-hand panels) and an $\mathcal{O}(100 \%)$ uncertainty at the jet level (right-hand panels). As with the fully leptonic system considered above, the large uncertainty is once again attributable to the parton-shower treatment of the forward jets using different scales.

We find that VBF cross sections with complicated kinematical cuts are extremely sensitive to the exact scale choice one uses, and it is insufficient to simply characterize the hard scattering with a rough estimate of $\mu$. While we have only considered the effects of varying $\mu$ on signal rates, the background is also susceptible to these uncertainties. Even without considering the effects of the veto cut, which can only increase sensitivity to $\mu$, it's natural to associate $\mathcal{O}(2 \times) K$-factors with high multiplicity QCD events. Therefore, before drawing any conclusion about the presence of new physics one would have to understand these systematics. In principle, the theoretical uncertainty may be reduced through higher order calculations that can give us a better idea of the appropriate scale choice. Substantial efforts would be needed both in theory and in experiments before to bring this uncertainty under control. With this in mind, in the next section we will present a new tool to circumvent the difficult issue of the factorization-scale dependence. 


\begin{tabular}{|c|c|}
\hline Pass conditions & Veto conditions \\
\hline$E\left(j_{\text {tag }}\right)>300 \mathrm{GeV}$ & $p_{T}\left(j_{\text {mini }}\right)>25 \mathrm{GeV}$ \\
$2<\left|y\left(j_{\text {tag }}\right)\right|<5$ & $\left|y\left(j_{\text {mini }}\right)\right|<2$ \\
$p_{T}\left(j_{\text {tag }}\right)>20 \mathrm{GeV}$ & $130 \mathrm{GeV}<m_{W J}<240 \mathrm{GeV}$ \\
$p_{T}\left(W_{\text {recon. }}\right)>320 \mathrm{GeV}$ & \\
$\left|y\left(W_{\text {had }}\right)\right|<4$ & \\
\hline
\end{tabular}

Table 3: $\quad W^{+} W^{-}$semi-leptonic decay cuts inspired by [19]. These require two tagged jets $\left(j_{\text {tag }}\right)$ and two reconstructed $W \mathrm{~s}\left(W_{\text {recon. }}\right)$. If the events contain two soft, central jets $\left(j_{\text {mini }}\right)$ they are vetoed. The cut on the jet- $W$ invariant mass is designed to reduce top quark backgrounds.

\begin{tabular}{|c|c|c|c|c|c|c|}
\hline & \multicolumn{3}{|c|}{ Parton Level [fb] } & \multicolumn{3}{c|}{ Jet Level [fb] } \\
\hline$c_{H} \xi$ & $\beta=0.5$ & $\beta=1.0$ & $\beta=2.0$ & $\beta=0.5$ & $\beta=1.0$ & $\beta=2.0$ \\
\hline 0.4 & 0.95 & 0.81 & 0.73 & 0.53 & 0.38 & 0.26 \\
0.2 & 0.82 & 0.72 & 0.64 & 0.43 & 0.33 & 0.24 \\
0.0 & 0.73 & 0.64 & 0.57 & 0.40 & 0.29 & 0.21 \\
\hline
\end{tabular}

Table 4: Cross sections [fb] for VBF with the semi-leptonic decay of $W^{+} W^{-}$for various anomalous Higgs-gauge couplings and at different factorization scales parameterized by Eq. (3.2). The cuts used to generate these results are those of Table 3 .

\section{Polarization Measurements}

With the uncertainties detailed above as our motivation, we propose a new technique to probe the anomalous couplings in a robust way. Our basic idea is to look for the relative increase in longitudinal vector boson production by comparing it to the production of transverse modes. Unlike the overall cross section, which is sensitive to the behavior of the forward jets, the relative transverse to longitudinal production rates should be stable against different scale choices because it depends only on the $V V \rightarrow V V$ scattering amplitude.

To measure the polarization of a vector boson we need to reconstruct the four-momenta of its decay products and measure their distribution with respect to a polarization axis. If one chooses the polarization axis to be the gauge boson direction of motion (Fig. 2), then a simple spin-analysis predicts that in the $V$ rest frame the transverse and longitudinal polarizations will be distributed as

$$
P_{ \pm}\left(\cos \theta^{*}\right)=\frac{3}{8}\left(1 \pm \cos \theta^{*}\right)^{2}, P_{L}\left(\cos \theta^{*}\right)=\frac{3}{4}\left(1-\cos ^{2} \theta^{*}\right)
$$

where $\theta^{*}$ denotes the angle between the parton and the gauge boson direction of motion in the gauge boson rest frame. 

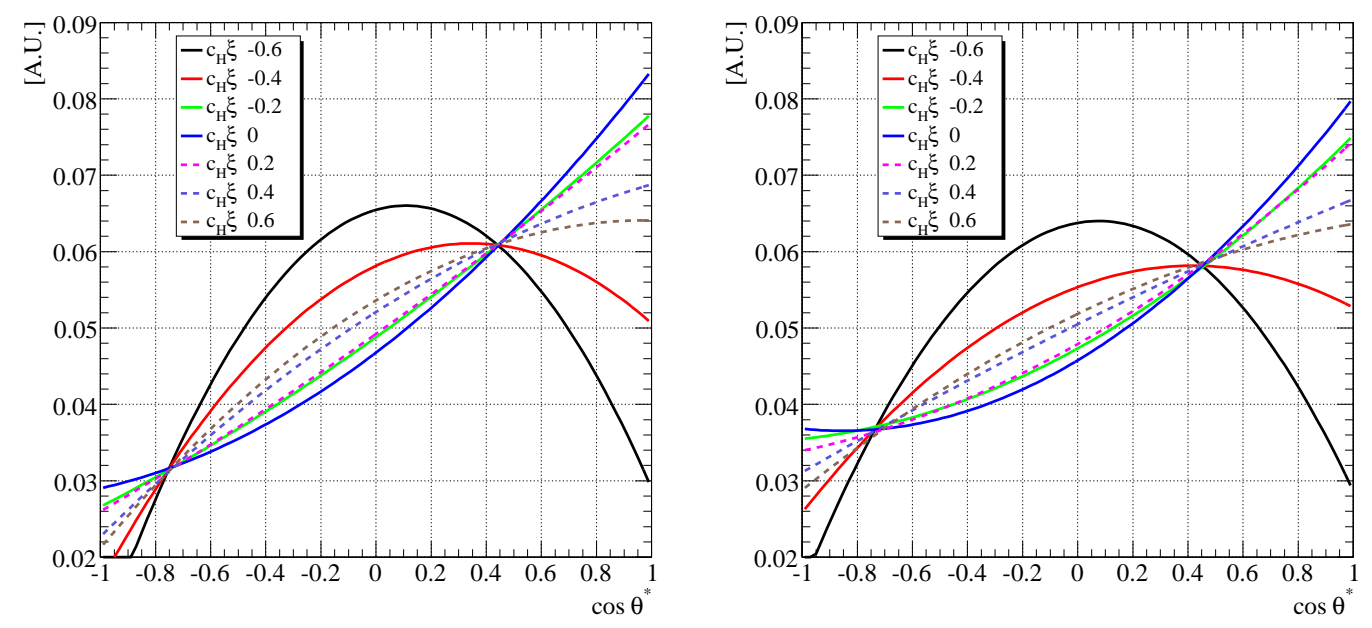

Figure 3: The distributions of $\cos \theta^{*}$ for different anomalous couplings at parton level (left) and for fully showered, hadronized, clustered, and reconstructed events (right). All distributions are normalized to the same area.

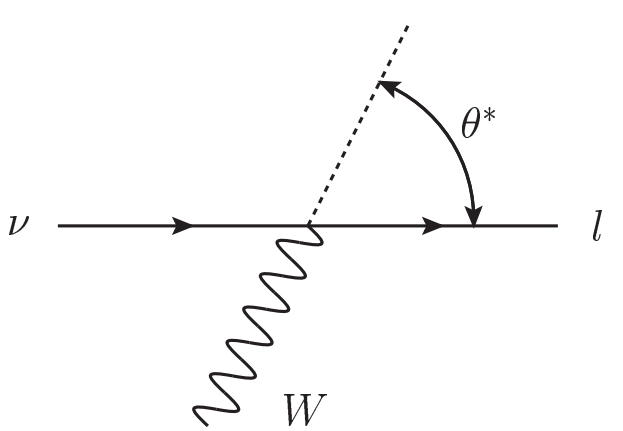

Figure 2: The polarization axis used to measure $\theta^{*}$. Note that this is measured in the rest frame of the $W$, and the $W$ direction of motion is defined with respect to the $W W$ center of mass.
To measure these distributions experimentally, we need to fully reconstruct the gauge boson pair center of mass and each gauge boson's direction of motion in this frame. To accomplish this we will focus on the semi-leptonic decay channel of the $V V$ system as this allows full reconstruction of the system while minimizing the SM background by requiring leptons and missing energy. The semi-leptonic channel also significantly increases the signal event rate. For this we will rely upon jet substructure techniques to reconstruct the hadronically decaying gauge boson [19]. We will focus on studying the $W^{+} W^{-}$final state, although we will take into account the background from other VBF processes like $W^{ \pm} W^{ \pm}$and $W^{ \pm} Z$ that enter because we can not distinguish the sign of a hadronically decaying vector, nor can we always distinguish a hadronically decaying $W$ from a $Z$. Later in this section we will comment on the $\operatorname{SM} \mathcal{O}\left(\alpha_{S}^{2}\right)$ and $\mathcal{O}\left(\alpha_{S}^{4}\right)$ backgrounds.

\subsection{Leptonic Polarization}

We begin with the polarization analysis for the leptonic side of the decay. We first study the parton-level results, then we will turn on the full simulation (parton-showering and jet clustering) to see that they are largely unchanged. The cuts we use are those in Table 3.

Before proceeding further, we encounter a subtlety in the reconstruction of the leptonic 


\begin{tabular}{|c|c|c|c|c|c|}
\hline & \multicolumn{2}{|c|}{ Leptonic $W$} & \multicolumn{2}{|c|}{ Hadronic $W$} & \\
\hline$c_{H} \xi$ & $f_{L}^{P}$ & $f_{L}^{J}$ & $f_{L}^{P}$ & $f_{L}^{J}$ & $\sigma[\mathrm{fb}]$ \\
\hline-0.6 & 0.77 & 0.74 & 0.71 & 0.55 & 3.38 \\
-0.4 & 0.58 & 0.52 & 0.49 & 0.40 & 1.12 \\
-0.2 & 0.33 & 0.30 & 0.23 & 0.24 & 0.60 \\
0.0 & 0.27 & 0.25 & 0.17 & 0.22 & 0.62 \\
0.2 & 0.34 & 0.31 & 0.24 & 0.26 & 0.65 \\
0.4 & 0.42 & 0.39 & 0.32 & 0.32 & 0.73 \\
0.6 & 0.46 & 0.42 & 0.40 & 0.38 & 0.87 \\
\hline
\end{tabular}

Table 5: The fraction of longitudinally polarized vector bosons for different anomalous couplings at parton level $f_{L}^{P}$ and jet level $f_{L}^{J}$, reconstructed in hadronic and leptonic decays. Also listed are the jet-level cross sections. These results are after the cuts of Table 3.

system: While the neutrino four-momentum is constrained by the on-shell $W$ condition, it is only determined up to a discrete ambiguity. One finds two candidate four-momenta at the same azimuthal angle but separated from the charged lepton by a fixed rapidity difference. In what follows we will simply use the average $\cos \theta^{*}$ value from both solutions as an approximation of the true value. This is acceptable because we are working in a boosted regime where the difference in rapidity between neutrino and lepton is small, making the curvature effects from the $(y, \phi)$ system sub-leading. The resultant distributions are shown in Fig. 3, at parton level (left panel) and after the hadronization (right panel). The characteristic shapes with different couplings are quite distinctive. In Table 5 we compute the cross section for each anomalous coupling and fit it to the transverse and longitudinal distributions of Eq. (4.1) using

$$
P\left(\cos \theta^{*}\right)=f_{L} P_{L}\left(\cos \theta^{*}\right)+f_{+} P_{+}\left(\cos \theta^{*}\right)+f_{-} P_{-}\left(\cos \theta^{*}\right)
$$

where the $P$ are normalized probability distributions of $\cos \theta^{*}$ and the $f$ are subject to the constraint $\sum f=1$. As one can see from comparing the jet and parton level figures, the results are remarkably stable under a full simulation.

In Figure 4 we plot the projected event distributions and associated statistical errors both for the SM and for an anomalous scenario with $c_{H} \xi=-0.4$, given $100 \mathrm{fb}^{-1}$ of luminosity. The shape difference between the two samples is clearly visible. To estimate the luminosity necessary to probe a given coupling, one can use that the signal scales roughly as $\left(c_{H} \xi\right)^{2}$, as discussed before. However, the precise reach of the LHC in discerning anomalous couplings will require a more thorough accounting of background (we discuss this further in Section 4.4). Further, we have not made an effort to optimize the statistical power of the analysis and there are other channels that contribute to the signal, such as $W^{+} W^{+}, W^{ \pm} Z$ and $Z Z$. In addition, one can extract more information from each event, as we will now see. 


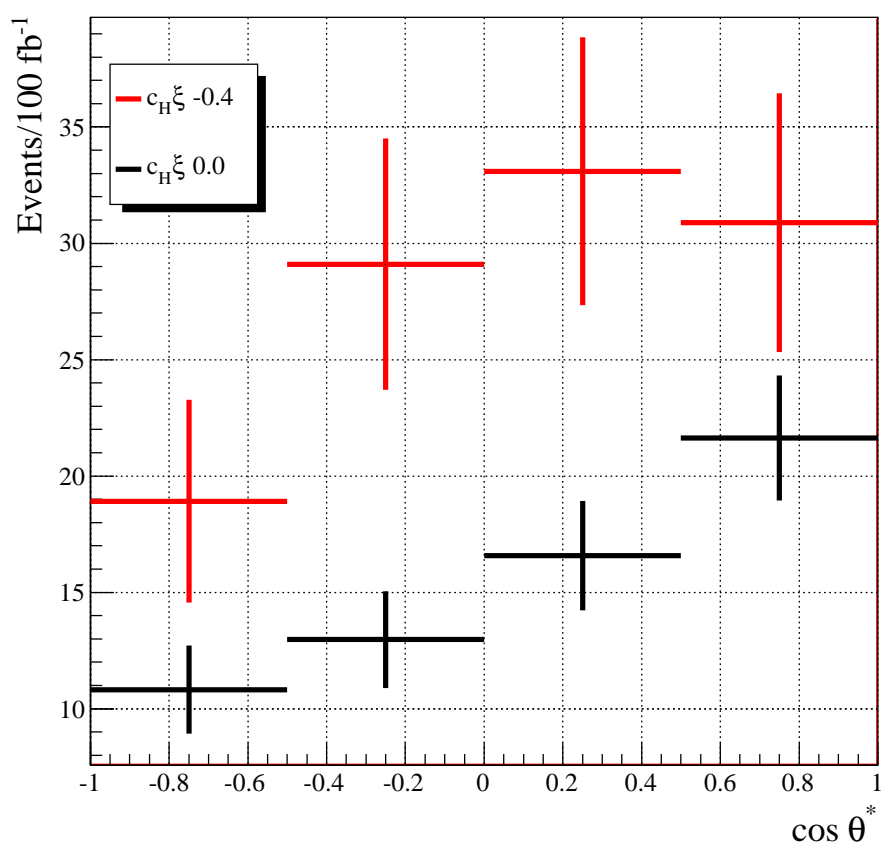

Figure 4: Projected distribution and associated statistical uncertainties of $\cos \theta^{*}$ for the leptonically decaying vector using $100 \mathrm{fb}^{-1}$ of luminosity.

\subsection{Hadronic Polarization}

It is possible to further improve the discriminating power of polarization by considering both sides of the $V V$ system together; by looking for the expected correlation between both states one can hope to gain additional discriminating power.

To see the correlation effect, consider Fig. 5, which shows the parton-level $\cos \theta^{*}$ distributions for both sides of the $V V$ system in SM and non-SM scenarios. For now, we plot $\cos \theta^{*}$ on the hadronic side for the down-type quarks. In the non-SM scenario we see a rapid rise in the central region of the plot near $\cos \theta^{*} \approx 0$. This indicates that the results are correlated; when we see a $V_{L}$ it is likely to be accompanied by a $V_{L}$ because only the $V_{L} V_{L}$ final state sees the $E^{2}$ growth characteristic of with non-SM effects. In practice the situation is slightly more complicated because we cannot label the light quark states once they shower and hadronize (e.g. we cannot distinguish a $u$ from a $d$ ), so the distributions we measure are symmetrized. However, the distributions still carry additional discriminating power, as one can see from the distributions in Fig. 6 and Fig. 7, and Table 5. Note that in fitting the symmetrized distributions we only fit to data from $0<\left|\cos \theta^{*}\right|<0.7$. In the regime where $\left|\cos \theta^{*}\right| \gtrsim 0.7$ one subject becomes very soft and the technique breaks down (although, of course, the leptonic analysis still works here).

To perform this analysis we had to look at the hadronically decaying $V$ using subjet techniques (for a short overview of jet algorithms and their behavior, see appendix B). In particular, we used the $k_{T}$ algorithm $[43,44]$ with $R=0.25$ to cluster the constituents of 

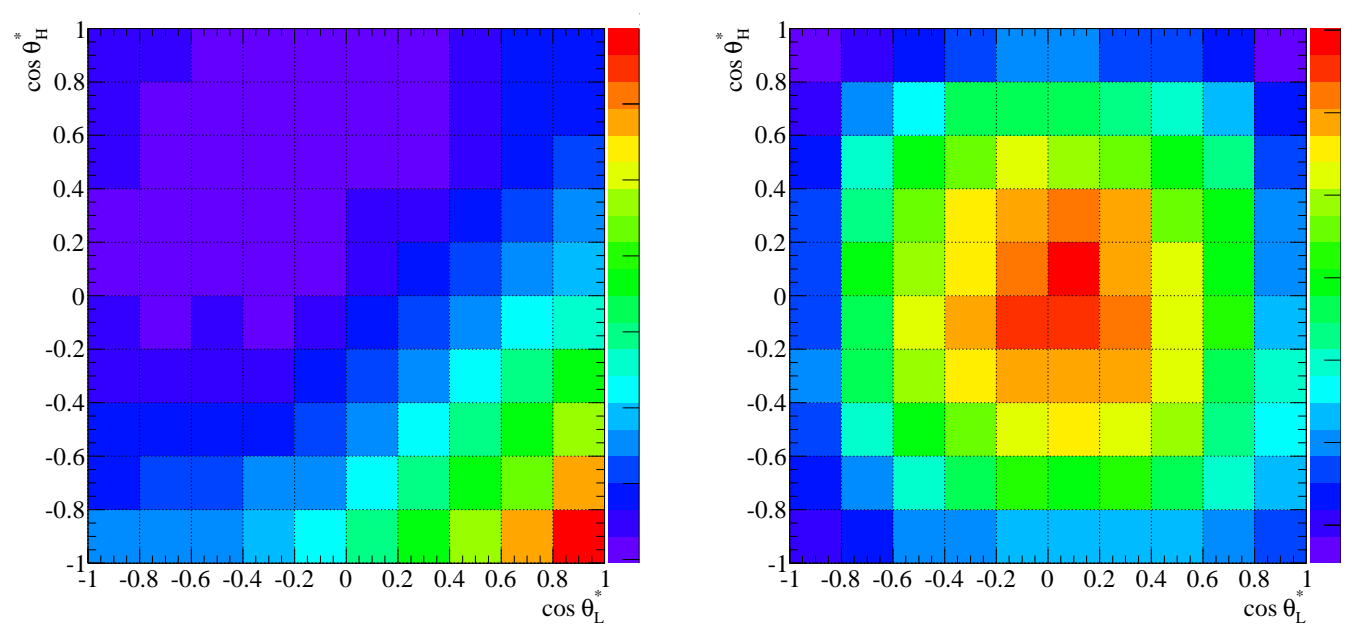

Figure 5: Distributions of $\cos \theta^{*}$ at parton-level for both sides of the $V V$ system (labeled with subscript $H$ and $L$ for hadronic and leptonic decays, respectively). The plot on the left is for the Higgs with SM couplings, while the one on the right is for $c_{H} \xi=-0.6$. The scale is individually normalized for each plot, going from violet to red as the concentration of events increases. The scaling of the color gradient on the right side of each plot is linear.

each hadronically decaying gauge boson, using the two most energetic subjets (as measured in the $V V$ center of mass frame) for our analysis. Note that rather than identifying our subjets through a $\mathrm{C} / \mathrm{A}[45,46]$ or $k_{T}$-like unwinding [47, 48], we used fixed small cones (i.e. small $R$ ). Otherwise, the subjets encompass a large area and become more susceptable to contamination from initial state radiation, multiple interactions, and event pileup. The choice of a small cone seems to result in a better reconstruction of events, especially at high values of $\cos \theta^{*}$ when there is a large difference in the subjet $p_{T} \mathrm{~s}$. Furthermore, we use $k_{T}$ rather than anti- $k_{T}$ to form our subjets because it more accurately reconstructs the softer jet in situations where the jets are nearly collinear (see appendix B).

One important thing to consider in the subjet analysis is that the results are not as robust in going from matrix-element to parton shower as were the leptonic results; the curves change shape (compare the parton and jet level results for both sides of the decay in Table 5). This is because the diffuse nature of the subjets makes them difficult to resolve when they become collinear and/or soft. We note, however, that at the LHC we can expect to calibrate subjet measurements for boosted hadronic $W$ s with large SM samples, and while the parton-level to jet-level results may vary, the correspondence should eventually be well understood. Thus the leptonic gauge boson analysis is likely to be the first tool used, but the hadronic analysis can be added later on.

\subsection{Scale Uncertainty}

We now explore the sensitivity of our results to the choice of the factorization scales. As before, we vary the scale choice as parameterized by Eq. (3.2). We present our fully showered results for the leptonically decaying $W$ in Table 6 using different scale choices. 

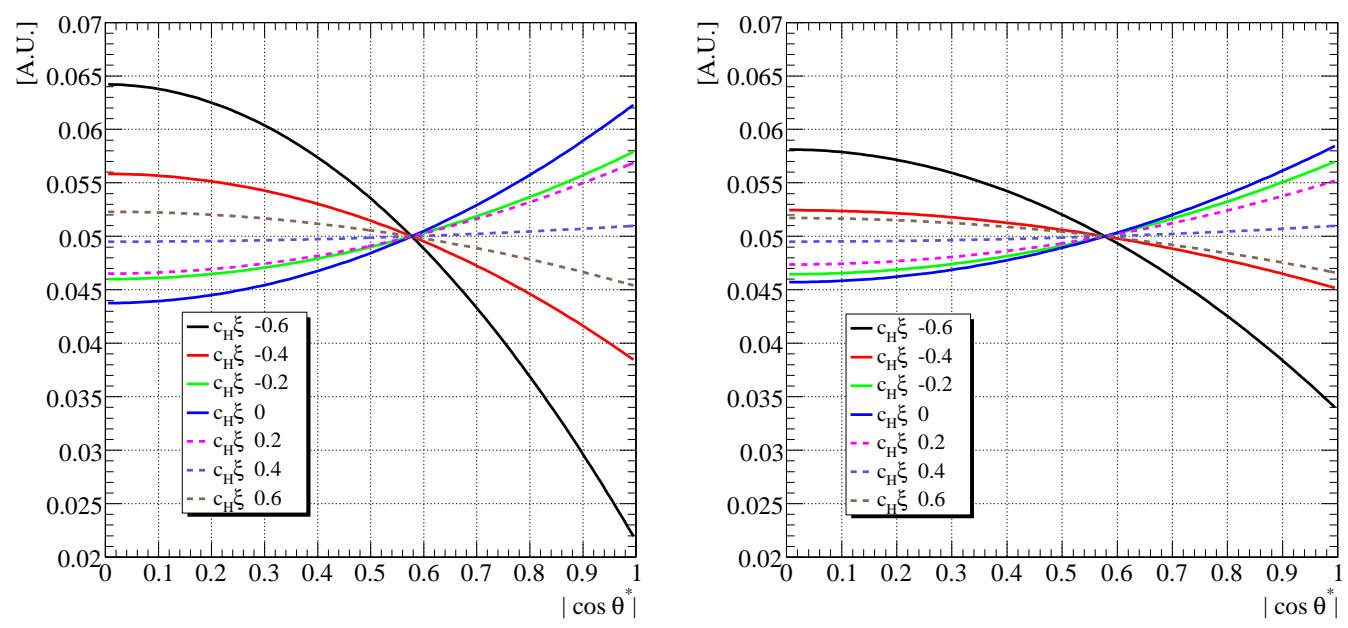

Figure 6: Distribution of $\left|\cos \theta^{*}\right|$ at different anomalous couplings for hadronically decaying $W \mathrm{~s}$ using parton level samples (left) and fully showered, hadronized samples (right). Note that the distributions differ more at high values of $\cos \theta^{*}$ because this is the region in which one jet is relatively soft.

\begin{tabular}{|c|c|c|c|}
\hline & \multicolumn{3}{|c|}{ Longitudinal Fraction } \\
\hline$c_{H} \xi$ & $\beta=0.5$ & $\beta=1.0$ & $\beta=2.0$ \\
\hline 0.0 & 0.25 & 0.26 & 0.25 \\
0.2 & 0.33 & 0.33 & 0.33 \\
0.4 & 0.40 & 0.40 & 0.41 \\
\hline
\end{tabular}

Table 6: The reconstructed fraction of longitudinally polarized leptonically decaying vector bosons measured for various anomalous couplings and at different factorization scales.

One can see from these results that the relative longitudinal to transverse fraction is a robust measure of anomalous couplings, steady across different values of $\beta$. The reason for this is clear: The showering and hadronization processes of the forward jets are independent of the behavior of the polarizations and decays of the gauge bosons. Whatever happens with the forward jet-tagging and the central jet-vetoing is isolated from the polarization measurements of the final decay products, hence the robust behavior.

\subsection{Background}

In our matrix element calculations, we have included the irreducible electroweak $V V j j$ backgrounds. A full treatment of the other background from QCD and top quarks is beyond the scope of this work, but here we give some rough estimates and discuss how background processes might have an effect upon the calculations. With the sophisticated acceptance cuts as adopted here, estimates of the background generally place it at or below the signal level. For the semi-leptonic modes of our interest in [19], whose cuts we mimic, 

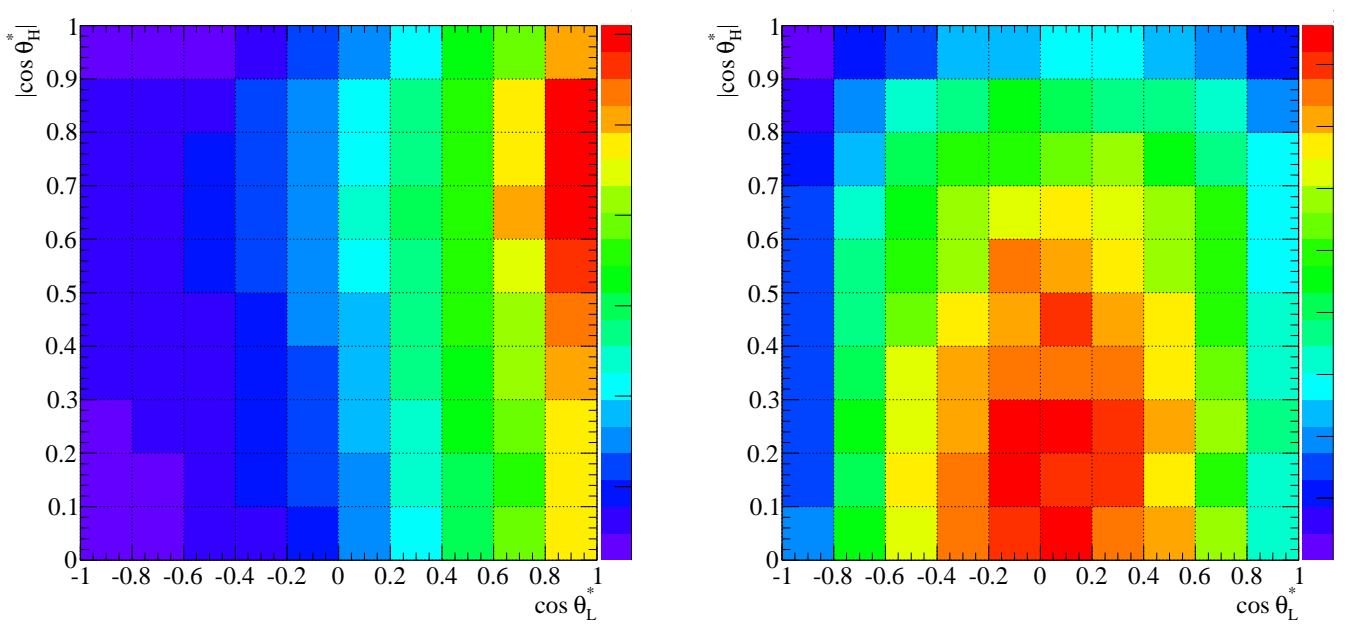

Figure 7: Jet-level distribution of $\cos \theta^{*}$ (labeled with subscript $H$ and $L$ for hadronic and leptonic decays, respectively) for the SM Higgs (left) and Higgs with $c_{H} \xi=-0.6$ (right). The scale is individually normalized for each plot, going from violet to red as the concentration of events increases. The scaling of the color gradient on the right side of each plot is linear.

the background is calculated to be roughly one quarter of the signal rate, so we expect the enhanced signal distributions to be discernible. We would like to emphasize that the robust feature of the polarization measurements should still persist when comparing with the background events, in which the leptonic decaying $W$ is mostly transversely polarized, and the di-jets from QCD have no particular angular preference. Thus further separation of the backgrounds may be possible by extending this technique to the two-dimensional angular plots where one looks for correlations as shown in the last section.

\section{Future Directions}

Here we discuss some further improvements one could make in reconstructing the hadronically decaying gauge bosons, along with some additional applications and extensions of our technique.

\subsection{Improving Hadronic Reconstruction}

Comparing the columns of Table 5, one can see that the polarization of the hadronically decaying gauge boson was reconstructed less accurately than the gauge boson that decayed leptonically. This is partly because at higher boosts the gauge boson decay products become more collinear and are consequently more difficult to resolve individually. While it is tempting to simply exclude this region from the analysis, the highly boosted region is precisely where we expect to see the greatest effects of new physics, so it is worthwhile to try to improve reconstruction in this regime.

One approach for better resolving the subjets would involve letting the subjet radius scale as $1 / p_{T}$, so that the size of the subjets is adjusted to naturally account for their 
kinematics. This sort of scaling was explored by [49], although in a non-subjet context. We note that it is likely one would have to adapt the $1 / p_{T}$ behavior to account for the finite calorimeter segmentation and crowded subjet environment if this approach were adopted.

Another approach, along a different line, would involve abandoning subjet construction at very high boosts in favor of an energy splitting technique. Such a technique would split the jet from a hadronically decaying gauge boson into two pieces and consider the degree of energy sharing between them using something akin to the $z$-variable of [48]:

$$
z=\min \left(E_{A}, E_{B}\right) / E_{\text {tot }}
$$

For $\cos \theta^{*} \approx 0$, where both daughters are transverse to the $V$ direction of motion we would expect $z \approx 0.5$, while for $\cos \theta^{*} \approx 1$ we expect $z \approx 0$. We avoided this technique earlier in our polarization analysis because it is not boost invariant, and an alternative definition of $z$ using $p_{T}$ instead of $E$ would still be sensitive to the orientation of the $V$ decay. However, these effects go as $m_{W} / p_{T}$, and so are marginalized as we consider higher boosts. Therefore, it might become advantageous to transition to a $z$ variable in the highest $p_{T}$ ranges.

\subsection{Additional Applications}

Throughout this article we have focused on scenarios with a light, SM-like Higgs because they can be very difficult to distinguish from the SM, making VBF analyses particularly useful. However, the VBF polarization measurements we have discussed could be of use in studying other models of EWSB.

Consider, for example, the class of models where $V_{L} V_{L}$ scatter amplitudes are unitarized by the exchange of new spin-1 states (see Fig. 8). Models in this class include the so-called Higgsless models, where higher dimensional gauge bosons unitarize the longitudinal amplitudes, and technicolor, which employs the technirho for this purpose $[50,51,52$, $53,54,55,56]$.

Phenomenological studies of these models $[57,58]$ often study the VBF production of the new resonances because the heavy spin-1 states involved in restoring unitarity must couple strongly to the longitudinal modes of the electroweak gauge bosons. While it would be striking to see a bump in the $V V$ invariant mass to reconstruct a resonance, seeing this correlated with a measured increase in the longitudinal production would give a true smoking gun signal. Furthermore, if the new states have a large width and can not easily be seen as a resonance, their presence might be inferred by a broad increase in longitudinal mode production near the particle mass. This would also be useful in probing a scenario with a heavy Higgs.

Furthermore, in models of EWSB where a heavy $Z^{\prime}$ mixes with the SM $Z$, there are even more dramatic effects one could look for. In these models the VBF aptitudes grow as $E^{4}$ up until the scale of the $Z^{\prime}$ [59]. These models can be difficult to probe if the $Z^{\prime}$ is too heavy to be produced, but one could in principle observe the unique amplitude growth characteristic of these scenarios.

Another extension of this sort of analysis using VBF as a robust probe of new physics would involve making use of azimuthal angle correlations. This sort of analysis has been 
performed with an eye toward distinguishing the spin of the unitarizing particle [60]. It would be interesting to see if this could yield an additional handle on background suppression or signal enhancement.

Finally, we note that while our article focuses on the $E^{2}$ growth in $V_{L} V_{L}$ amplitudes associated with new physics, for many interesting cases the rates are so low that with the LHC we can probably only hope to observe this growth when integrated over all higher energies. A luminosity upgrade to the LHC [61] might allow for the differential detection of this growth: given enough data, one could bin longitudinal fractions according to the $\hat{s}$ of the reconstructed $V V$ system, allowing for a differential detection of the amplitude growth.

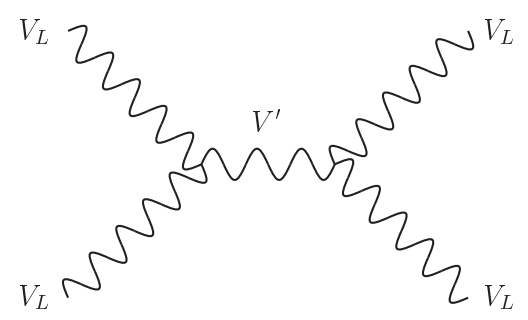

Figure 8: Unitarity restoration through a spin-1 particle.

\section{Conclusions}

In this article we have introduced a powerful new technique for identifying signs of beyond the SM physics associated with the EWSB by probing VBF processes at the LHC.

We began by motivating our decision to study models of EWSB employing a light Higg-like particle with couplings deviating from those of the SM. Theories with a light Higgs boson are favored by the current electroweak precision data. However, this type of model is the most difficult to distinguish from the SM, especially if the new physics particles are very heavy. However, it is also the scenario in which VBF can be most useful, because for such a scenario the amplitude for $V_{L} V_{L} \rightarrow V_{L} V_{L}$ scattering exhibits a non-SM $E^{2}$ growth until new physics comes into play.

Past analyses designed to measure this $E^{2}$ growth were reviewed and updated to account for the effects of the parton shower and jet clustering. While the cuts pioneered by these works can be very powerful in reducing the SM background, we demonstrate that there is still a significant $\mathcal{O}(100 \%)$ rate uncertainty attributable to factorization scale ambiguities. Thus, we show that in the absence of higher order calculations that might give us some guidance on the correct scale treatment, rate information alone may not be sufficient to distinguish the signs of new physics.

We then propose our new technique, which uses the semi-leptonic decay mode of the $V V$ system to fully reconstruct events and obtain the decay angle distributions for the $V$ daughters. These distributions can be decomposed into longitudinal and transverse components, allowing us to measure the $E^{2}$ growth in scattering amplitudes associated with new physics by looking for the relative increase in longitudinal production. We demonstrate that these results are insensitive to the scale ambiguities that trouble rate measurements.

In closing, we wish to reiterate that polarization measurements of VBF final states are a powerful, robust probe of new physics associated with the EWSB. Although we have only employed them here to study light SM-like Higgs scenarios, they would be useful in more general scenarios of EWSB as long as the longitudinal gauge bosons are significantly 
involved. Such measurements may prove to be our best tool in understanding the physics of EWSB at the LHC.

\section{Acknowledgments}

The authors would like to acknowledge useful discussions with Johan Alwall, Jiji Fan, Kentarou Mawatari, and Matt Schwartz. The work of T.H. is supported in part by the DOE under grants DE-FG02-95ER40896 and W-31-109-Eng-38, and in part by the Wisconsin Alumni Research Foundation. L.-T. W. was supported by NSF grant PHY-0756966 and DOE grant DE-FG02-90ER40542.

\section{A. Scattering Amplitudes for Longitudinal Gauge Bosons and Partial Wave Unitarity}

For completeness, we will here review the high energy behavior of longitudinal gauge boson scattering. This will demonstrate why we expect the increase in the scattering amplitudes for non-SM Higgs couplings. It will also help us establish the partial wave unitarity bound for longitudinal gauge boson scattering, which is of practical importance for our simulation. We will make use of the Goldstone equivalence theorem, which says that the scattering behavior of the longitudinal gauge bosons is the same as that of the eaten Goldstones, up to corrections of order $\mathcal{O}\left(m_{W} / E\right)$. Note that while we will only explicitly calculate the behavior of $W_{L}^{+} W_{L}^{-} \rightarrow W_{L}^{+} W_{L}^{-}$, the other longitudinal gauge boson scattering processes are similar.

We begin with the Lagrangian for the SM Higgs doublet with the additional dimension6 operator we wish to study:

$$
\mathcal{L}=\frac{1}{4} \operatorname{Tr}\left(\partial \mathcal{H}^{\dagger} \partial \mathcal{H}+\mu^{2} \mathcal{H}^{\dagger} \mathcal{H}\right)-\frac{\lambda}{16} \operatorname{Tr}\left(\mathcal{H}^{\dagger} \mathcal{H}\right)^{2}+\frac{c_{H}}{32 f^{2}}\left[\partial \operatorname{Tr}\left(\mathcal{H}^{\dagger} \mathcal{H}\right) \partial \operatorname{Tr}\left(\mathcal{H}^{\dagger} \mathcal{H}\right)\right]
$$

where $\mathcal{H}=\phi \cdot \sigma$ for real fields $\phi_{i}(i=0 \leftrightarrow 3)$ and $\sigma=(1, \vec{\sigma})$. The SM Higgs potential corresponds to $c_{H}=0$. Expanding around the minima $\left\langle\phi_{0}\right\rangle=\mu / \sqrt{\lambda}$ one finds new derivative interactions proportional to $c_{H}$. Those relevant to $\phi_{+} \phi_{-} \rightarrow \phi_{+} \phi_{-}$scattering at lowest order are:

$$
\mathcal{L} \supset-v \lambda h \phi_{+} \phi_{-}-\frac{\lambda}{2} \phi_{+}^{2} \phi_{-}^{2}+\frac{c_{H}}{2 f^{2}}\left(\phi_{+}^{2}\left(\partial \phi_{-}\right)^{2}+\phi_{+} \phi_{-} \partial \phi_{+} \partial \phi_{-}+2 v \phi_{+} \partial \phi_{-} \partial h\right)+\text { h.c. }
$$

where we have denoted the shifted $\phi_{0}$ field by $h$ and written $\phi_{1,2}$ in terms of their charge eigenstates $\phi_{ \pm}$. Also, note that there is an additional kinetic term for $h$ :

$$
\mathcal{L} \supset \frac{c_{H} v^{2}}{2 f^{2}}(\partial h)^{2}
$$

so that in going to canonical normalization we must insert a factor of $N=1 / \sqrt{1+c_{H} v^{2} / f^{2}}$ for every $h$ encountered at a vertex. The tree level amplitude becomes

$$
\mathcal{M}\left(\phi_{+} \phi_{-} \rightarrow \phi_{+} \phi_{-}\right)=-4 i \lambda+i \frac{c_{H}}{f^{2}} s-\frac{i N^{2}}{\left(s-m_{h}^{2}\right)}\left(2 \lambda v+\frac{c_{H} v}{2 f^{2}} s\right)^{2}+(s \leftrightarrow t)
$$


where $m_{h}=\sqrt{2 \lambda} v$. Working in the limit $s, t \gg m_{h}^{2}$ we find

$$
\mathcal{M}\left(\phi_{+} \phi_{-} \rightarrow \phi_{+} \phi_{-}\right) \approx i \frac{c_{H}}{f^{2}}\left(1-N^{2} \frac{c_{H} v^{2}}{4 f^{2}}\right)(s+t)
$$

which shows the $E^{2}$ growth in the amplitude that we expect. In this limit, the $J=0$ partial wave is:

$$
a_{0}=\frac{1}{16 \pi s} \int_{-s}^{0} \frac{c_{H}}{f^{2}}\left(1-N^{2} \frac{c_{H} v^{2}}{4 f^{2}}\right)(s+t) d t=\frac{c_{H} s}{32 \pi f^{2}}\left(1-N^{2} \frac{c_{H} v^{2}}{4 f^{2}}\right)
$$

Partial wave unitarity is violated when $\left|\operatorname{Re}\left(a_{I}\right)\right| \geq 1 / 2$, so the unitarity bound is saturated when

$$
s_{\max }=\frac{16 \pi v^{2}}{c_{H} \xi\left(1-c_{H} \xi N^{2} / 4\right)}
$$

To stay clear of this limit, we limit ourselves to studying events for which $s \leq 2 \mathrm{TeV}$ (corresponding to $\left|c_{H} \xi\right| \leq 0.6$ ).

We note that one may gain further intuition into the longitudinal gauge boson system by considering the parameterization

$$
\mathcal{H}=(v+h) e^{i \vec{\pi} \cdot \vec{\sigma} / v}
$$

Here we have shifted our field definitions so that the $\pi$ transform non-linearly. In this language, the relevant terms in the Lagrangian become

$$
\mathcal{L} \supset \frac{\sqrt{\lambda}}{\mu} h \partial \pi_{+} \partial \pi_{-}+\frac{\lambda}{6 \mu^{2}}\left(\pi_{+}^{2}\left(\partial \pi_{-}\right)^{2}-\pi_{+} \pi_{-} \partial \pi_{+} \partial \pi_{-}\right)+\text {h.c. }
$$

As before, the kinetic term of $h$ is shifted, so we must add a factor of $N$ at every point we encounter an $h$ at a vertex. Note, however, that in this case all of the operators come with $\partial \pi$ terms. Computed in this way, the amplitude $\mathcal{M}\left(\pi_{+} \pi_{-} \rightarrow \pi_{+} \pi_{-}\right)$shows the same behavior as Eq. (A.4), as it must, but this is the result of a non-cancelation of derivatives between the four-point operator and the $h$-exchange in the $t \& s$-channels, rather than because of a new vertex.

Using these results we can compare the scattering in the Higgsless case to that of the case where the Higgs has anomalous couplings. To consider the Higgsless case we set $c_{H}=0$ in Eq. (A.4) and consider the $\sqrt{s} \ll m_{h}$ limit using $m_{h}=\sqrt{2 \lambda} v$. We find

$$
\sigma_{\text {no-higgs }} \propto|\mathcal{M}|^{2}=\frac{4}{v^{4}}(s+t)^{2}
$$

wheras for the case of a light Higgs with anomalous couplings we find from Eq. (A.5)

$$
\sigma_{\text {anom-higgs }} \propto|\mathcal{M}|^{2}=\frac{\left(c_{H} \xi\right)^{2}}{v^{4}}(s+t)^{2}
$$

under the assumption

$$
\frac{c_{H} \xi}{2} \gg \frac{m_{h}^{2}}{s}
$$


This is how we arrived at Eq. (2.3). For $m_{h} \sim 100 \mathrm{GeV}$ this is true for scattering at the $\mathrm{TeV}$ scale as long as $c_{H} \xi \gtrsim 1 / 10$. At lower values of the anomalous coupling the dominant effect comes from interference effects proportional to $s+t$ instead of $(s+t)^{2}$. Thus smaller values of the anomalous couplings have a qualitatively different energy behavior that of larger values, making them especially difficult to resolve.

\section{B. Overview of Jet Algorithms}

While a comprehensive review of jet algorithms is beyond the scope of this work (see [62] for a recent review), here we will provide a short overview so the reader can quickly gain intuition into subjet techniques.

Jet algorithms can roughly be divided into two categories: cone algorithms, which function as cookie-cutters to stamp out jets from calorimeter cells, and sequential recombination algorithms, which build up a jet by merging four-momenta one by one in a prescribed order. Here we will focus on recombination algorithms.

Each of these algorithms functions by defining a distance measure between every pair of four-momenta and for each four-momenta individually:

$$
d_{i j}=\min \left(p_{T i}^{2 n}, p_{T j}^{2 n}\right)\left(\frac{R_{i j}}{R_{0}}\right)^{2}, d_{i B}=p_{T j}^{2 n}
$$

for jets $i$ and $j$. If the smallest distance measure at a given stage in clustering is between two four-momenta they are merged, otherwise the four-momenta with the smallest $d_{i B}$ is declared a jet and removed from the queue.

\begin{tabular}{|c|c|c|}
\hline Algortithm & $n$ & Approximate clustering order \\
\hline$k_{T}$ & 1 & soft $\rightarrow$ hard \\
C/A & 0 & near $\rightarrow$ far $($ in $y$ - $\phi$ ) \\
anti- $k_{T}$ & -1 & hard $\rightarrow$ soft \\
\hline
\end{tabular}

Table 7: Parameterization and approximate behavior of sequential recombination jet algorithms.

The different sequential recombination algorithms are distinguished by value of $n$ appearing in Eq. (B.1). These values determine the clustering order, whether one clusters beginning with hard four-momenta, soft four-momenta, or by angle (see Table 7 and Fig. 9). For the subjet analysis at hand, where reconstructing the softer subjet is essential, we therefore use the $k_{T}$ algorithm which begins by clustering softer jets, preventing them from being cannibalized by the harder subjet.

\section{References}

[1] Precision electroweak measurements on the $Z$ resonance, Phys. Rept. 427 (2006) 257, [hep-ex/0509008]. The ALEPH Collaboration, the DELPHI Collaboration, the L3 Collaboration, the OPAL Collaboration, the SLD Collaboration, the LEP Electroweak Working Group, the SLD electroweak, heavy flavour groups. 


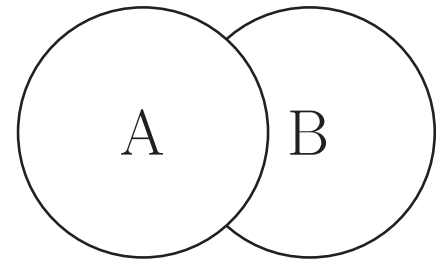

anti- $k_{T}$

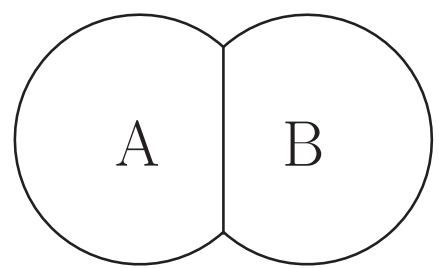

$\mathrm{C} / \mathrm{A}$

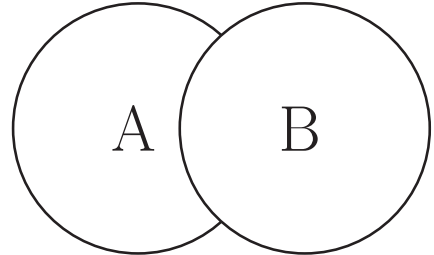

$k_{T}$

Figure 9: Approximate clustering behavior of jets for the different sequential recombination algorithms assuming $p_{T}^{A}>p_{T}^{B}$. Note that while we have shown the jets as being circular, the $k_{T}$ jets can behave in a non-circular, wandering way.

[2] R. Barbieri, A. Pomarol, R. Rattazzi, and A. Strumia, Electroweak symmetry breaking after LEP-1 and LEP-2, Nucl. Phys. B703 (2004) 127-146, [hep-ph/0405040].

[3] N. Arkani-Hamed, A. G. Cohen, and H. Georgi, Electroweak symmetry breaking from dimensional deconstruction, Phys. Lett. B513 (2001) 232-240, [hep-ph/0105239].

[4] R. Contino, Y. Nomura, and A. Pomarol, Higgs as a holographic pseudo-Goldstone boson, Nucl. Phys. B671 (2003) 148-174, [hep-ph/0306259].

[5] W. Buchmuller and D. Wyler, Effective Lagrangian Analysis of New Interactions and Flavor Conservation, Nucl. Phys. B268 (1986) 621.

[6] C. N. Leung, S. T. Love, and S. Rao, Low-Energy Manifestations of a New Interaction Scale: Operator Analysis, Z. Phys. C31 (1986) 433.

[7] J. Wudka, Electroweak effective Lagrangians, Int. J. Mod. Phys. A9 (1994) 2301-2362, [hep-ph/9406205].

[8] K. Hagiwara, S. Ishihara, R. Szalapski, and D. Zeppenfeld, Low-energy constraints on electroweak three gauge boson couplings, Phys. Lett. B283 (1992) 353-359.

[9] K. Hagiwara, S. Ishihara, R. Szalapski, and D. Zeppenfeld, Low-energy effects of new interactions in the electroweak boson sector, Phys. Rev. D48 (1993) 2182-2203.

[10] Particle Data Group Collaboration, K. Hagiwara et al., Review of particle physics, Phys. Rev. D66 (2002) 010001.

[11] V. Barger, T. Han, P. Langacker, B. McElrath, and P. Zerwas, Effects of genuine dimension-six Higgs operators, Phys. Rev. D67 (2003) 115001, [hep-ph/0301097].

[12] G. F. Giudice, C. Grojean, A. Pomarol, and R. Rattazzi, The Strongly-Interacting Light Higgs, JHEP 06 (2007) 045, [hep-ph/0703164].

[13] D. A. Dicus and V. S. Mathur, Upper bounds on the values of masses in unified gauge theories, Phys. Rev. D7 (1973) 3111-3114.

[14] B. W. Lee, C. Quigg, and H. B. Thacker, Weak Interactions at Very High-Energies: The Role of the Higgs Boson Mass, Phys. Rev. D16 (1977) 1519.

[15] M. J. G. Veltman, Second Threshold in Weak Interactions, Acta Phys. Polon. B8 (1977) 475.

[16] M. S. Chanowitz and M. K. Gaillard, The TeV Physics of Strongly Interacting W's and Z's, Nucl. Phys. B261 (1985) 379. 
[17] I. Low, R. Rattazzi, and A. Vichi, Theoretical Constraints on the Higgs Effective Couplings, 0907.5413.

[18] J. Bagger et al., CERN LHC analysis of the strongly interacting $W W$ system: Gold plated modes, Phys. Rev. D52 (1995) 3878-3889, [hep-ph/9504426].

[19] J. M. Butterworth, B. E. Cox, and J. R. Forshaw, WW scattering at the CERN LHC, Phys. Rev. D65 (2002) 096014, [hep-ph/0201098].

[20] J. Bagger, S. Dawson, and G. Valencia, Effective field theory calculation of $p p \rightarrow V(L) V(L)$ X, Nucl. Phys. B399 (1993) 364-394, [hep-ph/9204211].

[21] V. D. Barger, K.-m. Cheung, T. Han, and R. J. N. Phillips, Strong $W^{+} W^{+}$scattering signals at pp supercolliders, Phys. Rev. D42 (1990) 3052-3077.

[22] M. S. Chanowitz, Strong WW scattering at the end of the 90's: Theory and experimental prospects, hep-ph/9812215.

[23] D. A. Dicus, J. F. Gunion, and R. Vega, Isolating the scattering of longitudinal W+'s at the SSC using like sign dileptons, Phys. Lett. B258 (1991) 475-481.

[24] V. D. Barger, K.-m. Cheung, T. Han, J. Ohnemus, and D. Zeppenfeld, A Comparative study of the benefits of forward jet tagging in heavy Higgs production at the SSC, Phys. Rev. D44 (1991) 1426-1437.

[25] V. Hankele, G. Klamke, D. Zeppenfeld, and T. Figy, Anomalous Higgs boson couplings in vector boson fusion at the CERN LHC, Phys. Rev. D74 (2006) 095001, [hep-ph/0609075].

[26] J. Bagger et al., The Strongly interacting $W W$ system: Gold plated modes, Phys. Rev. D49 (1994) 1246-1264, [hep-ph/9306256].

[27] G. J. Gounaris, J. Layssac, and F. M. Renard, Vector boson pair production at supercollider: Useful approximate helicity amplitudes, Z. Phys. C62 (1994) 139-150, [hep-ph/9309324].

[28] A. Ballestrero, G. Bevilacqua, D. B. Franzosi, and E. Maina, How well can the LHC distinguish between the SM light Higgs scenario, a composite Higgs and the Higgsless case using VV scattering channels?, 0909.3838.

[29] K. Cheung, C.-W. Chiang, and T.-C. Yuan, Partially Strong WW Scattering, Phys. Rev. D78 (2008) 051701, [0803.2661].

[30] H.-J. He, Y.-P. Kuang, C. P. Yuan, and B. Zhang, Anomalous gauge interactions of the Higgs boson: Precision constraints and weak boson scatterings. ((U)), Phys. Lett. B554 (2003) 64-72, [hep-ph/0211229].

[31] B. Zhang, Y.-P. Kuang, H.-J. He, and C. P. Yuan, Testing anomalous gauge couplings of the Higgs boson via weak-boson scatterings at the LHC, Phys. Rev. D67 (2003) 114024, [hep-ph/0303048].

[32] R. N. Cahn, S. D. Ellis, R. Kleiss, and W. J. Stirling, Transverse Momentum Signatures for Heavy Higgs Bosons, Phys. Rev. D35 (1987) 1626.

[33] V. D. Barger, T. Han, and R. J. N. Phillips, Improving the Heavy Higgs Boson Two Charged Lepton-Two Neutrino Signal, Phys. Rev. D37 (1988) 2005-2008.

[34] R. Kleiss and W. J. Stirling, Tagging the Higgs, Phys. Lett. B200 (1988) 193.

[35] V. D. Barger, R. J. N. Phillips, and D. Zeppenfeld, Mini - jet veto: A Tool for the heavy Higgs search at the LHC, Phys. Lett. B346 (1995) 106-114, [hep-ph/9412276]. 
[36] R. N. Cahn and S. Dawson, Production of Very Massive Higgs Bosons, Phys. Lett. B136 (1984) 196.

[37] G. L. Kane, W. W. Repko, and W. B. Rolnick, The Effective W+-, Z0 Approximation for High-Energy Collisions, Phys. Lett. B148 (1984) 367-372.

[38] S. Dawson, The Effective W Approximation, Nucl. Phys. B249 (1985) 42-60.

[39] A. D. Martin, R. G. Roberts, W. J. Stirling, and R. S. Thorne, Physical gluons and high E(T) jets, Phys. Lett. B604 (2004) 61-68, [hep-ph/0410230].

[40] T. Sjostrand, S. Mrenna, and P. Skands, PYTHIA 6.4 physics and manual, JHEP 05 (2006) 026, [hep-ph/0603175].

[41] M. Cacciari, G. P. Salam, and G. Soyez, The anti- $k_{t}$ jet clustering algorithm, JHEP 04 (2008) 063, [0802.1189].

[42] M. Cacciari, G. Salam, and G. Soyez, "FastJet." http://fastjet.fr/.

[43] S. Catani, Y. L. Dokshitzer, M. H. Seymour, and B. R. Webber, Longitudinally invariant K(t) clustering algorithms for hadron hadron collisions, Nucl. Phys. B406 (1993) 187-224.

[44] S. D. Ellis and D. E. Soper, Successive combination jet algorithm for hadron collisions, Phys. Rev. D48 (1993) 3160-3166, [hep-ph/9305266].

[45] Y. L. Dokshitzer, G. D. Leder, S. Moretti, and B. R. Webber, Better Jet Clustering Algorithms, JHEP 08 (1997) 001, [hep-ph/9707323].

[46] M. Wobisch and T. Wengler, Hadronization corrections to jet cross sections in deep- inelastic scattering, hep-ph/9907280.

[47] J. M. Butterworth, A. R. Davison, M. Rubin, and G. P. Salam, Jet substructure as a new Higgs search channel at the LHC, Phys. Rev. Lett. 100 (2008) 242001, [0802.2470].

[48] J. Thaler and L.-T. Wang, Strategies to Identify Boosted Tops, JHEP 07 (2008) 092, [0806.0023].

[49] D. Krohn, J. Thaler, and L.-T. Wang, Jets with Variable R, JHEP 06 (2009) 059, [0903.0392].

[50] C. Csaki, C. Grojean, L. Pilo, and J. Terning, Towards a realistic model of Higgsless electroweak symmetry breaking, Phys. Rev. Lett. 92 (2004) 101802, [hep-ph/0308038].

[51] C. Csaki, C. Grojean, H. Murayama, L. Pilo, and J. Terning, Gauge theories on an interval: Unitarity without a Higgs, Phys. Rev. D69 (2004) 055006, [hep-ph/0305237].

[52] R. S. Chivukula, D. A. Dicus, H.-J. He, and S. Nandi, Unitarity of the higher dimensional standard model, Phys. Lett. B562 (2003) 109-117, [hep-ph/0302263].

[53] R. S. Chivukula et al., A three site higgsless model, Phys. Rev. D74 (2006) 075011, [hep-ph/0607124].

[54] Y. Nomura, Higgsless theory of electroweak symmetry breaking from warped space, JHEP 11 (2003) 050, [hep-ph/0309189].

[55] L. Susskind, Dynamics of Spontaneous Symmetry Breaking in the Weinberg- Salam Theory, Phys. Rev. D20 (1979) 2619-2625.

[56] S. Weinberg, Implications of Dynamical Symmetry Breaking: An Addendum, Phys. Rev. D19 (1979) 1277-1280. 
[57] A. Belyaev et al., Technicolor Walks at the LHC, Phys. Rev. D79 (2009) 035006, [0809.0793].

[58] A. Birkedal, K. Matchev, and M. Perelstein, Collider phenomenology of the Higgsless models, Phys. Rev. Lett. 94 (2005) 191803, [hep-ph/0412278].

[59] K. Cheung, C.-W. Chiang, Y.-K. Hsiao, and T.-C. Yuan, Longitudinal Weak Gauge Bosons Scattering in Hidden Z' Models, 0911.0734.

[60] K. Hagiwara, Q. Li, and K. Mawatari, Jet angular correlation in vector-boson fusion processes at hadron colliders, JHEP 07 (2009) 101, [0905.4314].

[61] W. Scandale and F. Zimmermann, Scenarios for sLHC and vLHC, Nucl. Phys. Proc. Suppl. 177-178 (2008) 207-211.

[62] G. P. Salam, Towards Jetography, 0906.1833. 